\title{
Stochastic GW searches and Cosmology with GWs
}

\author{
Giancarlo Cella* \\ Istituto Nazionale di Fisica Nucleare Sez. Pisa - Largo B. Pontecorvo 356127 Pisa Italy \\ E-mail: giancarlo.cella@pi.infn.it
}

\begin{abstract}
A stochastic background of gravitational waves can be described as a superposition of several uncorrelated contributions. It can be of both cosmological and astrophysical origin. In the first case, it can constitute potentially a unique probe of the primordial universe. In the second, it can give precious information on stellar populations. After discussing how this kind of signal can be detected and what information can be extracted from its study, I review the past and ongoing efforts to find it, the current upper limits and the future perspective for its detection.
\end{abstract}

The European Physical Society Conference on High Energy Physics

5-12 July

Venice, Italy

${ }^{*}$ Speaker. 


\section{Introduction}

A stochastic background of gravitational waves can be seen as a gravitational wave field which evolves from an initially random configuration, or as the result of a superposition of many uncorrelated and unresolved sources. There are potentially several kind of GW stochastic backgrounds, and accordingly with their origin they can be broadly classified as astrophysical and cosmological.

In the first case, the background's sources are astrophysical events (such as a compact binaries coalescence, a supernova, a rotating NS and so on) since the beginning of stellar activity. The study of an astrophysical SB can give important information about the evolution of stellar populations. Though a large number of potential sources have been studied, it is true that the predicted amplitudes are typically too small in order to be seen with current generation detectors. There are exceptions however, as we will see.

A cosmological SB carry in principle very important information about the early Universe, the reason being that gravitational interaction is so weak (compared, for example, with the electromagnetic one) that gravitational waves could be considered decoupled since the very beginning of the universe evolution. There are several mechanism that can generate a cosmological SB, and we will sketch briefly some of them. It must be admitted that also in this case the detection could be considered not guaranteed with current sensitivities. Once again there are interesting exceptions where the task does not seem hopeless. And the perspective for third generation earth-bound detectors and space ones like LISA seems quite interesting [1].

\section{The detection of a stochastic background}

The description of a stochastic background can be summarized by a mode expansion of the gravitational strain field in the frequency domain like

$$
\tilde{h}_{a b}(\vec{x}, \omega)=\sum_{P} \int d \Omega_{\hat{n}} \mathscr{A}_{\hat{n}, \omega}^{P} \varepsilon_{a b}^{P}(\hat{n}) e^{i \frac{\omega}{c} \hat{n} \cdot \vec{x}}
$$

Here each mode is parameterized by a polarization $P$ and a propagation direction $\hat{n}$, and its amplitude is represented by a stochastic variable $\mathscr{A}_{\hat{n}, \omega}^{P}$. The statistical properties of the stochastic background depend on the generation mechanism, and can be described by the cumulants of the $\mathscr{A}$ 's amplitudes.

A stochastic background can be safely considered stationary, because the typical physical time scales involved are much larger that the observation time. It is also expected to be typically Gaussian (but see Section 4), and in this case it is completely described by its second order cumulants. The coupling between the gravitational strain and a detector can be represented by a detector tensor $D^{a b}$, defined in such a way that the signal is given by $h_{I}=D_{I}^{a b} h_{a b}$. As a consequence form a statistical point of view the only relevant quantity is the second order correlation $\left\langle\tilde{h}_{I_{1}} \tilde{h}_{I_{2}}\right\rangle$ between the signal of a pair of detectors.

The optimal detector can be obtained starting from the specific form of this correlation. As an example, if the stochastic background is also isotropic and non polarized it comes out that the optimal statistic is given by

$$
Y=\int d \omega \frac{\tilde{x}_{1}^{*}(\omega) \tilde{x}_{2}(\omega) \gamma_{12}(\omega) S_{h}(\omega)}{S_{1}(\omega) S_{2}(\omega)}
$$


where $S_{i}$ is the power spectrum of the $i$-th detector noise, $S_{h}$ the theoretical power spectrum of the stochastic background and $\tilde{x}_{i}$ the output of the $i$-th detector, which is espected to be a sum of the signal $\tilde{h}_{i}$ and of the noise. The function $\gamma_{12}$ is called overlap reduction function, and is equal to the spectral coherence between the signals coupled to the two detectors. A loss of coherence is expected, since two detectors are coupled in a different way to the modes of the expansion (2.1), unless they are in the same place and oriented in the same way. In particular, a loss of coherence for the signal at a frequency $f$ is expected when the separation $d$ between the two detectors become of the same order or larger than the corresponding wavelength, $f>c d^{-1}=\tau^{-1}$. For example the separation between the two LIGO detectors if about $10^{-2}$ light-seconds, and the coherence is reduced by $50 \%$ around $50 \mathrm{~Hz}$. Equation (2.2) tell us that in order to detect a stochastic background the output of two different detectors must be correlated. The Signal to Noise Ratio can be defined as

$$
\mathrm{SNR}^{2} \propto T \int_{0}^{\infty} S_{h}^{2}(\omega) \frac{\gamma_{12}^{2}(\omega)}{S_{1}(\omega) S_{2}(\omega)} d \omega
$$

and as expected it is reduced by a loss of coherence. This means that it is good for a pair of detectors to be aligned, and in principle it should be good for tem to be one near the other. However it must be taken into account that the analysis which leads to the optimal statistic (2.2) is based on the hypothesis that the noise of the two detectors is uncorrelated, and it is difficult to be sure about that if they are nearby. Note also the proportionality SNR $\propto \sqrt{T}$ which tell us that the probability of detection increase with the observation time, though not particularly fast.

\section{Sources, sensitivities and upper limits.}

A prediction for the amplitude of a stochastic background can be represented by its differential energy spectrum, which is defined as a energy density for logarithmic interval of frequency normalized to the critical energy density, and is connected to the observed power spectrum of the gravitational strain signal by

$$
h_{0}^{2} \Omega_{G W}(f)=\frac{1}{\rho_{c}} \frac{d \rho_{g w}}{d \log f}=\frac{4 \pi^{2} h_{0}^{2}}{3 H_{0}^{2}} f^{3} S_{h}(f)
$$

Probably the most fundamental production mechanism for a cosmological GW stochastic background is the parametric amplification. Basically it can be understood by looking at the effect of the time dependence of the scale parameter $a$ in a Friedmann-Robertson-Walker metric which describes an expanding universe. The evolution of the amplitudes of GW modes in (2.1) is given by an equation like

$$
\ddot{\mathscr{A}}_{\omega}+\left(\frac{\omega^{2}}{c^{2}}-\frac{\ddot{a}}{a}\right) \mathscr{A}_{\omega}=0
$$

which describes an harmonic oscillator with a time dependent parameter. The transition between two different evolution regime, for example between the inflationary epoch and the radiation dominated one, can produce an amplification of the amplitudes. However the predicted final background predicted in the standard inflationary scenario is too small to be detected with the current and foreseen sensitivities [7] (see blue line in Figure 1). Non standard models for universe evolution can produce more interesting results. Alternative cosmologies like pre-big bang scenarios (red line in 


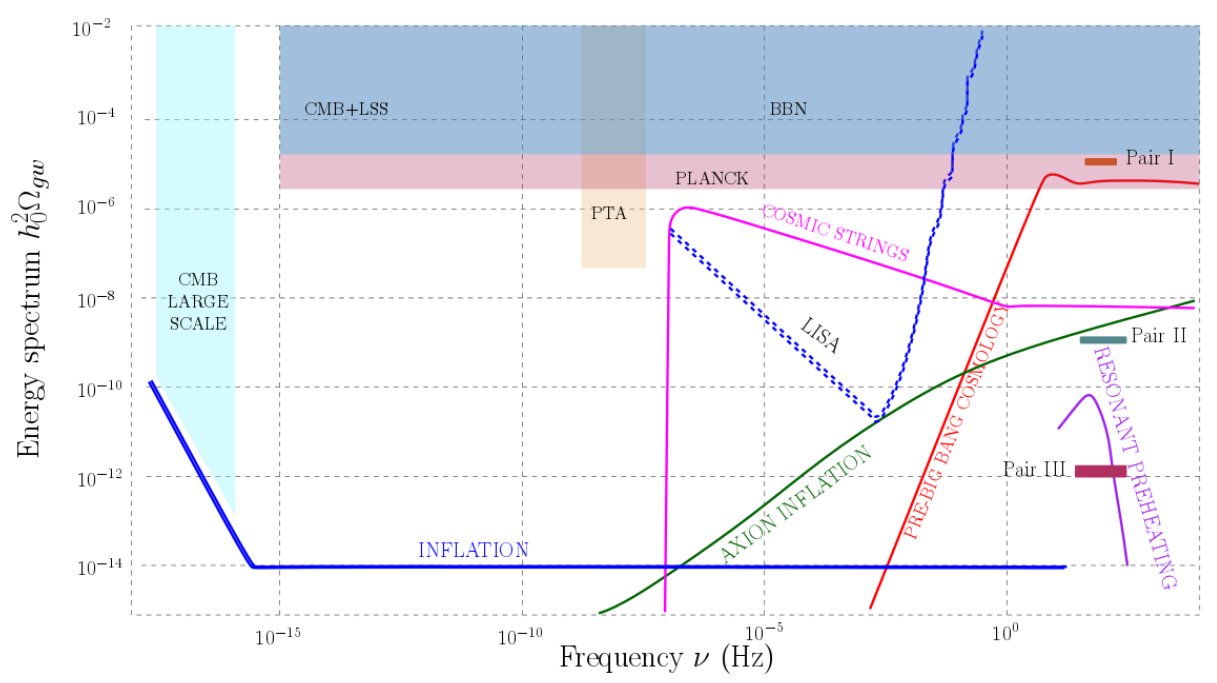

Figure 1: Some expected spectra of cosmological GW stochastic backgrounds, compared with upper limits and current sensitivities. Continuous lines correspond to energy spectrum predicted for a selection of production mechanism, and are described in the text. Dashed lines correspond to the sensitivity level which can be obtained using few months of data with a pair of first (LIGO, VIRGO) second (LIGO Advanced, VIRGO Advanced [2]) and third (ET [3]) generation detectors, and with LISA. Filled area represent current upper limits. BBN is an integral bound obtained from the observed abundances of light elements [4]. CMB bounds are related to cosmic microwave background observations $[4,5]$. PTA is obtained from pulsar timing array observations [6].

Figure 1) can evade the CMB large anisotropy upper limit at lower frequency and the BBN-CMB integral bound at higher ones, and can produce a significant background at the frequencies of interest for earth-bound detectors [8]. In inflation models which include couplings between axion and gauge fields [9] (green line in Figure 1) the gauge field back-reaction on the inflaton can extend the inflationary epoch, in such a way that GW production at higher frequencies is increased. During a resonant preheating phase at the end of the inflation, inflaton energy can be transferred efficiently to other particles. This mechanism can also be described by Equation (3.2), and the prediction for this kind of backgorund can be interesting for third generation detectors (purple line in Figure 1). Spectrum peak depends on energy scale (which is choosen at $10 \mathrm{GeV}$ in the plot, higher frequencies correspond to a larger scale).

A completely different production mechanism is connected with the presence of a dynamical network of cosmic (super)strings. String entering in the horizon can evolve dynamically, and a population of string loops can be generated via interconnections. These loops can emit gravitational waves, especially when cusps and kinks are present, and generate a stocastic background similar to the one represented in Figure 1 (magenta line) [10]. There is a large parameter space to esplore for these models, and observations started to give constraint about it.

I will not discuss here astrophysical backgrounds, and the interested reader can refer to reviews [11]. Interestingly, as a byproduct of the recent direct observation of gravitational waves [1] it has been possible to give a quantitative prediction about the expected astrophysical backgound generated by a population of binary $\mathrm{BH}-\mathrm{BH}$ coalescences in the observed mass range. It comes out that such a background could be detected with the current generation of advanced detectors [12]. 
Until now there is no evidence of a stochastic background in the data of the LIGO-VIRGO collaboration, however interesting upper limits exists [13], which correspond to constraint on models' parameters. The analysis of the last data taken during the "first detection" run of LIGO is in progress, and at least a large improvement of these limit is expected.

\section{Conclusions and perspectives}

Earth-bound GW detector measurements started to set interesting upper limits and constraints on models, especially when combined with other observations [14]. These limits are expected to improve when the data coming from the LIGO and VIRGO advanced detectors will be analyzed, and accordingly with theoretical predictions in some particular cases a detection could be possible.

When the sensitivity will further improve, for example with third generation detectors such as ET [3], which is expected to gain an order of magnitude in strain compared with advanced detectors, several more refined investigations will become of interest. In the case of a detection it will be possible to estimate parameters and disentangle contributions coming from different polarizations. This will be particularly interesting to test for models beyond general relativity. These tipically predict additional polarizations with respect to the standard plus and cross one. Data analysis techniques that make possible these studies are known [15] and currently applied to the available data. It will be also possible to test for anisotropies, using techniques which are also well defined and applied in the LIGO-VIRGO collaboration [16]. Finally, it will be possible to test non Gaussian models [17]. Many astrophysical stochastic backgrounds can potentially be non Gaussian, and in some cases (for example cosmic strings) this can be true for cosmological backgrounds. The reason for this is that the background can be generated by a number of events (for example compact binaries coalescences) whose signals overlap in such a way that it is not possible to separate the single contribution. But the number of overlapped events can be not very large, and in this case the central limit theorem cannot be applied. The optimal detection of these non Gaussian backgrounds is a non trivial problem, and interesting information about the model can be in principle extracted. This is an open issue.

In all the three cases mentioned (polarization disentanglement, study of anisotropies, study of non gaussianities) the availability of an extended network of detectors is crucial in order to apply the relevant data analysis techniques. Advanced VIRGO will join soon the two LIGO detectors, increasing the number of detector pairs by a factor three. In the near future also KAGRA and LIGO India will join, opening good perspectives for the study of a kind of signal which is not the most easy to detect, but can reward us with a lot of very relevant physical information.

\section{References}

[1] Ligo Scientific Collaboration and Virgo Collaboration collaboration, B. P. Abbott, R. Abbott, T. D. Abbott, M. R. Abernathy, F. Acernese, K. Ackley et al., Observation of gravitational waves from a binary black hole merger, Phys. Rev. Lett. 116 (Feb, 2016) 061102.

[2] B. P. Abbott, R. Abbott, T. D. Abbott, M. R. Abernathy, F. Acernese, K. Ackley et al., Prospects for observing and localizing gravitational-wave transients with advanced ligo and advanced virgo, Living Reviews in Relativity 19 (Feb, 2016) 1. 
[3] M. Punturo, M. Abernathy, F. Acernese, B. Allen, N. Andersson, K. Arun et al., The einstein telescope: a third-generation gravitational wave observatory, Classical and Quantum Gravity 27 (2010) 194002.

[4] M. Maggiore, Gravitational wave experiments and early universe cosmology, Physics Reports 331 (2000) $283-367$.

[5] I. Sendra and T. L. Smith, Improved limits on short-wavelength gravitational waves from the cosmic microwave background, Phys. Rev. D 85 (Jun, 2012) 123002.

[6] Z. Arzoumanian, A. Brazier, S. Burke-Spolaor, S. J. Chamberlin, S. Chatterjee, B. Christy et al., The nanograv nine-year data set: Limits on the isotropic stochastic gravitational wave background, The Astrophysical Journal 821 (2016) 13.

[7] M. S. Turner, Detectability of inflation-produced gravitational waves, Phys. Rev. D 55 (Jan, 1997) R435-R439.

[8] V. Mandic and A. Buonanno, Accessibility of the pre-big-bang models to ligo, Phys. Rev. D 73 (Mar, 2006) 063008.

[9] N. Barnaby, E. Pajer and M. Peloso, Gauge field production in axion inflation: Consequences for monodromy, non-gaussianity in the cmb, and gravitational waves at interferometers, Phys. Rev. D 85 (Jan, 2012) 023525.

[10] X. Siemens, V. Mandic and J. Creighton, Gravitational-wave stochastic background from cosmic strings, Phys. Rev. Lett. 98 (Mar, 2007) 111101.

[11] T. Regimbau, The astrophysical gravitational wave stochastic background, Research in Astronomy and Astrophysics 11 (2011) 369.

[12] Ligo Scientific Collaboration and Virgo Collaboration collaboration, B. P. Abbott, R. Abbott, T. D. Abbott, M. R. Abernathy, F. Acernese, K. Ackley et al., Gw150914: Implications for the stochastic gravitational-wave background from binary black holes, Phys. Rev. Lett. 116 (Mar, 2016) 131102.

[13] Ligo and Virgo Collaboration collaboration, J. Aasi, B. P. Abbott, R. Abbott, T. Abbott, M. R. Abernathy, T. Accadia et al., Improved upper limits on the stochastic gravitational-wave background from 2009-2010 ligo and virgo data, Phys. Rev. Lett. 113 (Dec, 2014) 231101.

[14] P. D. Lasky et al., Gravitational-wave cosmology across 29 decades in frequency, Phys. Rev. X6 (2016) 011035, [1511.05994].

[15] A. Nishizawa, A. Taruya, K. Hayama, S. Kawamura and M.-a. Sakagami, Probing nontensorial polarizations of stochastic gravitational-wave backgrounds with ground-based laser interferometers, Phys. Rev. D 79 (Apr, 2009) 082002.

[16] Ligo Scientific Collaboration and Virgo Collaboration collaboration, J. Abadie, B. P. Abbott, R. Abbott, M. Abernathy, T. Accadia, F. Acernese et al., Directional limits on persistent gravitational waves using ligo s5 science data, Phys. Rev. Lett. 107 (Dec, 2011) 271102.

[17] S. Drasco and E. E. Flanagan, Detection methods for non-gaussian gravitational wave stochastic backgrounds, Phys. Rev. D 67 (Apr, 2003) 082003. 